Review

\title{
Genetics, Toxicity, and Distribution of Enterohemorrhagic Escherichia coli Hemolysin
}

\author{
Maike Schwidder, Laura Heinisch and Herbert Schmidt *(D) \\ Department of Food Microbiology and Hygiene, Institute of Food Science and Biotechnology, \\ University of Hohenheim, Garbenstr. 28, 70599 Stuttgart, Germany \\ * Correspondence: Herbert.Schmidt@uni-hohenheim.de
}

Received: 6 August 2019; Accepted: 27 August 2019; Published: 29 August 2019

\begin{abstract}
The ability to produce enterohemolysin is regarded as a potential virulence factor for enterohemorrhagic Escherichia coli (EHEC) and is frequently associated with severe human diseases such as hemorrhagic colitis (HC) and the hemolytic uremic syndrome (HUS). The responsible toxin, which has also been termed EHEC-hemolysin (EHEC-Hly, syn. Ehx), belongs to the Repeats in Toxin (RTX)-family of pore-forming cytolysins and is characterized by the formation of incomplete turbid lysis zones on blood agar plates containing defibrinated sheep erythrocytes. Besides the expression of Shiga toxins (Stx) and the locus of enterocyte effacement (LEE), EHEC-Hly is a commonly used marker for the detection of potential pathogenic E. coli strains, although its exact role in pathogenesis is not completely understood. Based on the current knowledge of EHEC-Hly, this review describes the influence of various regulator proteins, explains the different mechanisms leading to damage of target cells, discusses the diagnostic role, and gives an insight of the prevalence and genetic evolution of the toxin.
\end{abstract}

Keywords: Escherichia coli hemolysin (EHEC-hemolysin, EHEC-Hly); Escherichia coli (EHEC); Shiga toxin-producing E. coli (STEC); repeats in toxin (RTX); enterohemolytic phenotype; virulence factor

Key Contribution: This review focusses on the current knowledge on genetics, function, and detection of EHEC-hemolysin of STEC strains.

\section{Introduction}

In early studies on the production of hemolysins in pathogenic Escherichia coli strains, Beutin et al. described that enteropathogenic E. coli demonstrated only a weak hemolytic activity when compared with extraintestinal E. coli strains [1]. They could also show that the hemolysin-encoding genes of E. coli serotypes O114 and O126 strains were encoded on the chromosome, but those of O26 strains were encoded on a large plasmid [1]. Later on, the authors found this weak hemolytic phenotype frequently in enteropathogenic E. coli (EPEC) strains isolated from infants with diarrhea. Therefore, they introduced the term enterohemolysin [2]. The authors characterized the enterohemolytic phenotype by the morphology of the zones of lysis on blood agar plates containing defibrinated sheep erythrocytes. Whereas the typical clear $\alpha$-hemolytic zone appeared after $3 \mathrm{~h}$ of growth at $37^{\circ} \mathrm{C}$, the "enterohemolytic" zones of enteropathogenic E. coli O26 strains appeared biphasic after overnight incubation. This was characterized by a clear zone beneath and a small turbid zone around the colony, which looks like an incomplete hemolysis [2]. These studies were extended to Verotoxin-positive E. coli strains and Beutin and colleagues found a close association of enterohemolysin and Verotoxin (Shiga toxin)-production [3]. Figure 1 shows the characteristic enterohemolytic phenotype of the enterohemorrhagic E. coli (EHEC) O157:H7 strain EDL933 in comparison to the lysis zones of the 
$\alpha$-hemolysin-producing UPEC strain U4-41, STEC O113:H21 strain TS18/08 as well as its deletion and complementation mutants, and laboratory strain $\mathrm{DH} 5 \alpha$ which is not able to produce hemolysin.



Figure 1. Different hemolysis zones on a blood agar plate containing defibrinated sheep erythrocytes. (A) E. coli Dh5 $\alpha$ is non-hemolytic, (B) EHEC O157:H7 strain EDL933 shows the typical enterohemolytic phenotype, (C) strain U4-41 shows the typical $\alpha$-hemolytic phenotype, (D) wildtype STEC O113:H21 strain TS18/08 demonstrates the typical enterohemolytic phenotype, (E) its isogenic $\Delta h n s$ mutant is hyper-hemolytic with large and clear zones of hemolysis, (F) and its complemented derivative TS18/08 $\Delta h n s+h n s$ is enterohemolytic. Production of blood agar plates, hemolytic phenotypes, and control strains have been previously described $[3,4]$.

Stroeher and Beutin later described enterohemolysin 1 (Ehly1) and Ehly2-DNA sequences which were located on temperate bacteriophages in Shiga toxin-negative E. coli $\mathrm{O} 26: \mathrm{H}^{-}$strains. By molecular techniques, it has been shown that the EHly-1 and Ehly-2 sequences were not present in all hemolytic E. coli strains, suggesting the presence of different hemolysins [5]. The frequency of enterohemolytic/enterohemorrhagic $E$. coli strains and the fact that the enterohemolytic phenotype was lost in a plasmid-cured derivative of an E. coli O157:H7 strain were the reasons to investigate the nature of the enterohemolysin production in EHEC strains in more detail [6]. In an early study, we could show that the enterohemolytic phenotype of E. coli O157:H7 strain EDL 933 was encoded on its large virulence plasmid pO157 and that it was not based on the presence of Ehly-1 and Ehly-2-sequences. Moreover, the enterohemolytic phenotype could be transferred by transformation of plasmid pO157 to laboratory strain E. coli HB101. DNA fragments of pO157 were subcloned, respectively, that carried the genetic information for EHEC hemolysin (EHEC-Hly) production [6]. Subsequent studies were then carried out to uncover the genetics of EHEC-Hly production in enterohemolytic E. coli strains (see below).

\section{Genetics of EHEC-Hemolysin}

Typical EHEC strains are able to express a number of virulence factors which are encoded on prophages, pathogenicity islands, or large plasmids. Among these, the expression of Shiga toxins (Stx) and the pathogenicity island termed "locus of enterocyte effacement" (LEE) are regarded as the most important virulence factors and are associated with severe humane diseases. Whereas the Shiga toxins, which belong to the class of $\mathrm{AB}_{5}$ toxins, exert a cytotoxic effect on Vero cells and HeLa cells [7], 
the LEE pathogenicity island is essential for the formation of so called "attaching and effacing" (A/E) lesions in the intestinal mucosa. These are characterized by the local loss of microvilli, and an intimate attachment of the bacteria to the enterocytes, together with the formation of pedestal-like structures on the apical membrane [8,9]. In addition, the ability to produce EHEC-Hly is regarded as another potential factor contributing to bacterial virulence $[10,11]$.

As mentioned before, Schmidt et al. have shown that EHEC-hlyA (syn. ehxA) of EHEC O157:H7 strain EDL933 is located on the large virulence plasmid pO157 [6]. Sequence analysis of pO157 demonstrated two open reading frames with about $60 \%$ homology to the $h l y C$ and $h l y A$ genes of the E. coli $\alpha$-hemolysin $(\alpha$-hlyA) operon [12]. Therefore, they were termed EHEC hlyA and EHEC hlyC to show the relationship to the $\alpha-h l y$ genes but also to distinguish them from. Further in silico analysis of the predicted amino acid sequence of EHEC-hlyA revealed 13 tandemly repeated sequences at the $\mathrm{C}$-terminus as well as the presence of a hydrophobic domain in the amino $\mathrm{N}$-terminal region, which was shown to be involved in pore formation of E. coli $\alpha$-HlyA [13]. Because of these similarities to the E. coli $\alpha$-hly operon, the putative EHEC-Hly was also assigned to the RTX-family of pore-forming cytolysins [12]. In a subsequent study, Schmidt and colleagues further analyzed the large plasmid pO157 by sequencing the DNA region downstream of the EHEC-hlyC and EHEC-hlyA genes [14]. Similar to the $\alpha$-hly operon in E. coli, two further genes were discovered and termed EHEC-hlyB and EHEC-hlyD, respectively. Altogether the four genes are located on the EHEC-hly operon with the gene order CABD similar to that of other proteins of the RTX-family [14]. While EHEC-hlyA is encoding a 107-kDa protein, which functions as the structural gene for EHEC-Hly, the EHEC-hlyC gene product, is assumed to be involved in posttranslational modification of the toxin, similar to $\alpha$-HlyC of E. coli $[12,15]$. The genes encoding EHEC-HlyB and EHEC-HlyD, in turn, share high sequence similarities with other RTX transport proteins, such as $h l y B$ and $h l y D$ of $E$. coli, which are required for transport of the toxin out of the bacterial cell $[14,15]$.

In a study of Boerlin et al. [16], the diversity of EHEC-hlyA in various Shiga toxin-producing E. coli (STEC) of different origins, as well as the relationship of EHEC-hlyA to the genomic background of STEC was examined. The results obtained by sequencing and Restriction Fragment Length Polymorphism (RFLP) analysis showed that EHEC-hlyA is highly conserved among a wide range of STEC serotypes, with approximately less than $4 \%$ of nucleic acid substitutions at the maximum [16]. Furthermore, phylogenetic analysis of a subset containing 27 STEC isolates, one enteropathogenic $E$. coli isolate, and a $\mathrm{K}-12$ reference isolate revealed that STEC isolates, positive for the LEE-marker gene eae, all evolved out of a single evolutionary lineage. Moreover, the results showed that the large plasmid carrying EHEC-hlyA has developed within this lineage, independently of recent horizontal transfer. In addition, two major groups of plasmids (type I and II) carrying the EHEC-hly genes were described that were supposed to differed considerably regarding the presence of other virulence-associated factors, as the LEE pathogenicity island seems to be present only in a single phylogenetic lineage harboring the type I plasmid [16]. These findings were later confirmed by studies of Cookson et al. and Newton and colleagues $[17,18]$. Using PCR and RFLP analysis, Cookson and colleagues performed phylogenetic analysis of EHEC-hlyA in E. coli strains from different sources (animal and human). According to sequence similarities of the different EHEC-hlyA alleles identified, STEC strains could also be divided into two groups, containing eae-positive and eae-negative strains. Additionally, a third group of eae-positive, st $x$-negative E. coli (atypical enteropathogenic E. coli strains) isolates were analyzed by Cookson et al. (a group which was not included in the previous study of Boerlin et al.), but these strains could all be assigned to the eae-positive STEC strains according to their EHEC-hly $A$ sequences. Furthermore, the authors were able to describe six genetically distinct EHEC-hlyA subtypes (A to F) [17]. Newton et al. investigated and compared the genetic background and relationship of the large plasmids pO113 of LEE-negative STEC strain O113:H21 and pO157 of LEE-positive STEC O175:H7 strain. By allelic profiling of the EHEC-hlyA gene, it was demonstrated that the two plasmids belong to different evolutionary lineages, confirming the results obtained from Boerlin et al., and Cookson et al. 
mentioned above. Moreover, the virulence plasmids of LEE-negative STEC strains were found to be highly related [18].

\section{Regulation of EHEC-hlyA Expression}

In Escherichia coli O157:H7 strain EDL933, transcription of the EHEC-hly operon is influenced by several regulators as it is included in the complex regulation circuit of the LEE pathogenicity island.

Three regulators, Ler, GrlR, and GrlA are encoded within the LEE, whereas Ler acts as a global activator for expression of most of the LEE genes $[19,20]$. In turn, the global regulator of LEE activator GrlA and the global regulator of LEE repressor GrlR are positive and negative regulators, controlling the transcription of the ler gene [21]. During a study with deletion mutants in EHEC O157:H7, Saitoh et al. showed that grlR deletion significantly increased the lytic activity of EHEC-Hly on plates containing defibrinated sheep erythrocytes, whereas in a $\Delta g r l A \Delta g r l R$ double mutant no such effect was observed [22]. Furthermore, the expression of EHEC-hlyA was considerably increased by overexpression of GrlA using a multicopy plasmid. This effect could even be demonstrated even in a ler deletion mutant, and led the authors conclude that GrlA plays a crucial role in the upregulation of EHEC-hlyA genes. Subsequent studies on the regulation of EHEC-hlyA gene expression by Iyoda and colleagues demonstrated, that the global LEE regulator Ler also acts as an activator for EHEC-hly $A$ expression in a GrlA-independent way and even more was shown to bind directly to the regulatory region of EHEC-hlyC in gel mobility shift assays [23]. In the same study, the non-LEE encoded LysR-type regulator LrhA, which positively regulates the expression of LEE genes [24], was shown to activate the EHEC-hly $A$ expression, even in a $\Delta l e r \Delta g r l A$ double deletion mutant. Moreover, it was demonstrated that LrhA also activates EHEC-hlyC gene expression by direct binding to the regulatory region. See Figure 2 for a schematic overview of influences and effects among different regulators on EHEC-hly expression.


Figure 2. Schematic representation of EHEC-hlyCABD regulation. Regulators with positive effect on EHEC-hly expression are indicated in green boxes, regulators with negative effect in red boxes. Influences among the different regulators, with regard to EHEC-hlyCABD expression, are indicated in green arrows for positive influence or red arrows for negative influence, respectively. Arrows facing the regulatory region of EHEC-hlyCABD are indicating a direct influence by binding of the regulator protein (not in scale to the actual binding point). 
During another study with EHEC O157:H7 strain EDL933, Li et al. investigated the influence of the histone-like nucleoid-structuring protein H-NS, the $\sigma$ factor RpoS, and DsrA on EHEC-hlyCABD expression [25]. The small regulatory RNA DsrA is known to inhibit H-NS function and to enhance RpoS synthesis [26,27]. Thus, DsrA was shown to be involved in the regulation of many virulence factors, such as the expression of genes within the LEE [28]. The results of Li et al. indicated that DsrA influences transcription of the EHEC-hly operon in a positive manner by two different temperature dependent ways: During cultivation at $30^{\circ} \mathrm{C}$, overexpression of DsrA increased EHEC-hlyA transcription in the wild-type EHEC O157:H7 strain, but not in the hns deletion mutant. In contrast, at $37^{\circ} \mathrm{C}$, DsrA overexpression stimulated EHEC-hlyA transcription in both the wildtype and the hns deletion mutant. Therefore, the authors assumed that DsrA might influence transcription of the EHEC-hlyCABD operon by a H-NS-dependent way at $30^{\circ} \mathrm{C}$ and in an H-NS-independent way at $37^{\circ} \mathrm{C}$. The global transcription regulator H-NS was demonstrated to decrease EHEC-hlyCABD expression at $30^{\circ} \mathrm{C}$, whereas no significant influence was shown during growth at $37^{\circ} \mathrm{C}$, and was therefore supposed to be responsible for temperature dependent control of EHEC-hlyA expression. The results of this study indicated further that the $\sigma$ factor RpoS, a known mediator of the general stress response in E. coli [29] plays a crucial and primary role in the regulation of EHEC-hlyCABD expression, as no levels of EHEC-hlyA mRNA could be detected in a rpoS deletion mutant, independent of growth temperature or DsrA overexpression. As it was shown before that RpoS concentration was increased in an hns deletion mutant [30], the authors assumed that H-NS might influence the expression of EHEC-hlyCABD either direct by binding to the upstream regulatory region or indirect by decreasing the expression of RpoS (for an overview see Figure 2). Interestingly, contrary results, regarding the global negative regulator H-NS, were obtained by a study of Rogers et al. [31], in which a deletion of the hns gene in STEC O91:H21 strain B2F1 led to a hyper-hemolytic phenotype. Moreover, using electrophoretic mobility shift assays, they could demonstrate that H-NS binds to an $88 \mathrm{bp}$ upstream region of the EHEC-hly operon and thus functions as direct negative regulator of EHEC-hlyCABD transcription. The authors assumed that the LEE-negative STEC strains used in the study might probably be the reason for the discrepancy to the study of Li et al., who examined the influence of H-NS in a LEE-positive EHEC O157:H7 strain [31]. This assumption is further supported by the results obtained in our group, in which a deletion of $h n s$ in the eae-negative STEC O113:H21 strain TS18/08 (first described in [32]) was constructed for the purpose of another research project concerning subtilase cytotoxin expression [33]. The hns deletion mutant showed a hyper-hemolytic phenotype on blood agar plates, whereas the complementation of the mutant with a recombinant $h n s$ gene on a medium-copy plasmid restored the characteristic wild-type turbid lysis zones. Figure 1 shows the hemolytic phenotypes of different $E$. coli strains. The hyper-hemolytic phenotype of strain TS18/08 $\Delta h n s$ can clearly be distinguished from the enterohemolytic phenotype of the wildtype strains. The large clear zone of hemolysis indicates an upregulation of the EHEC-hly operon.

\section{Function of EHEC-Hemolysin and Interaction with Host Cells}

Schmidt et al. demonstrated earlier that EHEC-hlyA encodes a functional RTX-toxin with a similar pore-forming capacity as the $\alpha$-Hly of E. coli [34]. In experiments with lipid bilayer membranes, EHEC-Hly formed transient ion-permeable channels with a median diameter of $2.6 \mathrm{~nm}$, which is similar to the channel-formation of E. coli $\alpha$-Hly described by Bhakdi and colleagues [35]. Through the insertion of the toxin into the eukaryotic membrane, a change in permeability is caused and resulting in the physical damage of the target cell. Because of the sequence similarities between EHEC-Hly and $\alpha$-Hly of E. coli and the comparable pore size it was suggested that both toxins might function in an identical way. However, differences in the toxic effect on cultured human and bovine were determined. As mentioned before, the studies from Schmidt et al. showed that, EHEC EDL 933 produced very small zones of hemolysis on blood agar plates and showed no hemolytic activity in culture supernatants, which is different to $\alpha$-Hly of E. coli [12]. However, with functioning EHEC-hlyBD genes supplied in trans, the hemolysis zones were visible much clearer and a hemolytic activity could be measured in 
the culture supernatants. Thus, it was assumed that the incomplete lysis might be due to a defective secretion machinery in EHEC strain EDL933. Similar results were obtained by Bauer and Welch [15]. In addition, their results indicated some target cell specificity of EHEC-Hly. Although it did not show an significant lytic effect on two lymphoma cell lines of human origin, complete lysis was observed for BL-3 cells (cells from a bovine lymphoma cell line) and sheep and human erythrocytes [15].

Taneike et al. demonstrated that nonpathogenic E. coli HB101 induced the production of interleukin-1 $\beta$ (IL-1 $\beta$ ) in human monocytes, after transformation of a recombinant plasmid carrying the EHEC-hly operon of an EHEC strain isolated from patient samples [36]. Similar results were obtained in a study of Zhang et al. (2012), in which EHEC-Hly induced the production of IL-1 $\beta$ in human macrophages and was also shown to contribute to cytotoxicity of these cells [37]. As it is known that synthesis of the Stx-receptor $\mathrm{Gb}_{3}$ is increased by IL-1 $\beta$ [38], it was speculated, that EHEC-Hly may contribute to bacterial virulence by enhancing the effect of Shiga toxin (Stx) [36]. Additionally, cytotoxicity to human brain microvascular endothelial cells (HBMECs) could be demonstrated for the EHEC-Hly of Stx-negative E. coli O26 strains isolated from patients with hemolytic-uremic syndrome (HUS). The authors therefore suggested, that EHEC-Hly might enhance the pathogenicity of Stx-negative E. coli O26 strains and may contribute to the development of HUS [39].

It has already been assumed, that EHEC-Hly remains associated mostly to the cell surface, for unclear reasons [12,14]. Aldick and colleagues investigated the grade of EHEC-Hly secreted extracellularly, and found the toxin in two forms: A free, soluble one and one associated to outer membrane vesicles (OMVs) released by EHEC during growth [40], reviewed in [41]. As it is known that many Gram-negative bacteria produce OMVs, which function as vehicle for the transport of toxins and other molecules (reviewed in [42]), Aldick et al. investigated the role of OMVs during EHEC-Hly release. The results of this study showed that EHEC-Hly is associated with OMVs in its biologically active form. Though this association, the toxin was considerably more stable under physiological conditions and showed prolonged hemolytic activity, as compared to free EHEC-Hly. Furthermore, immunogold staining of OMVs containing EHEC-Hly demonstrated that the toxin is located on the exterior surface. For both free and OMV-associated EHEC-Hly, the hemolytic activity was shown to depend strongly on the presence of calcium, a condition which is identical to other members of the RTX toxin family [43]. Additionally, it was demonstrated that the initial binding of OMVs to target erythrocytes is mediated by EHEC-Hly and that this binding step is also calcium-dependent [40]. Since free and soluble EHEC-Hly is known to lyse human microvascular endothelial cells most probably through pore formation in the cell membranes [34,39], the interaction of OMV-associated EHEC-Hly with human microvascular endothelial cells was examined in a subsequent study [44]. By using a model with human brain microvascular endothelial cells (HBMEC) and Caco-2 cells, it was shown that OMV-associated EHEC-Hly did not lyse the target cells via pore formation but triggers apoptotic cell death. The OMV-associated toxin was shown to be first internalized into the target cells via endocytosis and then translocated into endo-lysosomes. Due to endosomal acidification, EHEC-Hly is separated from the OMV and then translocated to mitochondria, resulting in a decrease of the mitochondrial transmembrane potential and cytochrome c release. Through the subsequent activation of caspase- 9 and caspase-3, the intrinsic apoptotic pathway is triggered, which leads to death of the host cells [44]. Interestingly, the internalization into host cells via OMVs, and subsequent triggering of the intrinsic apoptotic pathway, was also shown for Shiga toxin 2a (Stx2a) and the cytolethal distending toxin $\mathrm{V}$ $(\mathrm{CdtV})$, two major virulence factors of EHEC O157. This indicates the importance of the interactions of EHEC-Hly with OMVs for bacterial pathogenicity [45].

\section{Detection of EHEC-Hemolysin}

During an early study of Beutin et al. [3], various STEC strains isolated from human and animals were analyzed for the presence EHEC-Hly by molecular and microbiological methods. Because of the close association of the production of EHEC-Hly and Stx, EHEC-Hly was proposed as a marker for the rapid detection of potential pathogenic E. coli strains [3]. In a subsequent study, Beutin et al. [46] 
established a system for rapid identification and isolation of STEC from human stool by combining the EHEC-Hly test on blood agar plates with the "reverse passive latex agglutination test for the detection of verocytotoxins (Stx) 1 and 2" (VTEC-RPLA), as a rapid detection system for Stx and EHEC-Hly production. Using this combination, pathogenic STEC strains could be isolated from stool and characterized for toxin production within 72 to $96 \mathrm{~h}$ [46].

To improve the nonselective enterohemolysin agar described by Beutin et al. [3], Lehmacher and colleagues [47] modified this medium by adding vancomycin, cefixime, and cefsulodin to the blood agar medium (BVCC). Thus, the growth of non-E. coli bacteria present in fecal and food samples was inhibited and a competing hemolysis of Enterobacteriaceae and Gram-positive bacteria was prevented. After a brief pre-enrichment in mTSB (modified tryptic soy broth) for $6 \mathrm{~h}$ and subsequent overnight culturing on BVCC agar, $92.6 \%$ of the 95 investigated STEC strains were shown to produce EHEC-Hly. In contrast, only $80 \%$ of the same analyzed strains formed hemolysis zones on enterohemolysin agar. Therefore, the authors recommended this method for a reliable isolation and identification of STEC strains from stool and food samples [47].

In a study of Schmidt and Karch [10], a number of 36 STEC O111 strains, isolated from patients suffering from HUS or diarrhea, were characterized for the presence and production of EHEC-Hly. Whereas the enterohemolytic phenotype on blood agar plates was observed in $88 \%$ of the samples from patients with HUS, this phenotype could be demonstrated only in $22.2 \%$ isolates from patients with diarrhea. Furthermore, two strains showed a nonhemolytic phenotype, although the EHEC-hlyA gene was detected by DNA-based methods. Therefore, a combination of Stx-determination and EHEC-Hly characterization was recommended for routine screening of STEC O111 and other non-O157 STEC strains, similar to the results shown by Beutin et al. [46]. The high incidence of EHEC-Hly in isolates from patients with HUS, in contrast to patients suffering from diarrhea, led the authors suggest that EHEC-Hly might increase the capability of the bacteria to cause extraintestinal complications in humans [10].

Different results were obtained in a study of Bai et al., in which stool samples of patients with diarrhea HUS were tested for serogenotypes, stx subtypes as well as the presence of the intimin gene eae and EHEC hlyA [48]. In total, 75 Stx-positive isolates were analyzed, of which 59 isolates carried the EHEC-hlyA gene and 35 harbored eae. Interestingly, almost all of the eae positive isolates, except one, were also positive for EHEC-hlyA. The presence of eae and EHEC-hlyA appeared to be associated with bloody diarrhea, whereas only one of the three HUS isolates carried the EHEC_hlyA gene and none of them was positive for eae. These results indicate that the correlation of various STEC virulence factors and their involvement in causing clinical symptoms is very complex and remains to be elucidated. Moreover, during analysis of more than 300 STEC strains isolated from patients in Denmark, it was demonstrated that approximately 77\% carried EHEC-hlyA. However, on the basis of multivariate analysis the EHEC-hlyA gene did not play a significant role for the development of severe disease like HUS or bloody diarrhea [49].

Although the role of EHEC-Hly in bacterial pathogenicity is still not completely understood, the toxin is meanwhile regarded as a potential virulence factor and commonly used marker for the characterization of STEC strains. For example, EHEC-hlyA is one of the genetic markers tested in the U.S. Food and Drug Administration Escherichia coli Identification (FDA-ECID) microarray, which provides rapid characterizing of STEC isolates [50]. By using the FDA-ECID microarray, Shridhar et al. [51] analyzed six STEC O104:H7 strains isolated from cattle feces to determine their virulence profiles and to compare the profiles with that of human O104:H7 strains (clinical), the STEC strain O104:H4 (German outbreak strain), and STEC strain O104:H21 (milk-associated Montana outbreak strain). Interestingly, all investigated strains, both bovine and human isolates, were negative for the LEE marker gene eae. In contrast, EHEC-hlyA was present in four of the six bovine strains, in all human O104:H7 strains, with one exception, and in the Montana outbreak strain (O104:H21). Because all bovine O104:H7 strains were positive for st $x 1$ and EHEC-hlyA, as well as genes coding for various adhesins, this serotype was regarded as a possible foodborne pathogen in humans [51]. 


\section{Prevalence and Correlation with Other Virulence Factors}

Although EHEC-hly genes are found in many STEC serotypes that are associated with diarrheal disease and HUS, the precise role of the respective toxin in the pathogenesis of EHEC is still not completely understood. For example, Gyles at al. [52] investigated the association of the EHEC-hly $A$ gene with different STEC serotypes and found, that EHEC-hly $A$ was more frequently detected in in serotypes which are commonly implicated in disease (89\%) than in serotypes that are less frequently involved in disease (46\%), indicating an association of EHEC-Hly and pathogenesis of STEC. On the other hand, more than 1000 E. coli isolates from healthy cattle were analyzed, showing that the EHEC-hlyA gene was only present in 12 independent strains, out of which only 3 strains were also positive for st $x$. Therefore, they concluded that the presence of EHEC-hly $A$ alone is not significant enough for the detection of pathogenic STEC in bovine feces, as the EHEC-hlyA gene is present in non-STEC isolates as well [52]. In contrast, Sandhu et al. [53] showed in a subsequent study, using a total of 422 STEC samples isolated from cattle, that 153 samples $(\sim 36 \%)$ were positive for EHEC-hly $A$ and moreover, that the toxin was present in nearly all of the eae-positive isolates (98\%) and only in $34 \%$ of eae-negative isolates. Furthermore, EHEC-hlyA was highly associated with serotypes involved in human disease and thus was suggested as a marker for virulence of bovine STEC in humans. However, the authors concluded that EHEC-hlyA should not be used to predict the potential virulence of an isolate, as it is also present in STEC which are not implicated in human disease [53].

In a study of Slanec et al. [32] the virulence profiles of various STEC strains, isolated from risk foods such as minced meat or raw milk products, were characterized. The EHEC-hlyA gene was one of the most abundant virulence markers detected. Interestingly, EHEC-hlyA was found in 30 out of 75 investigated strains $(\sim 40 \%)$, whereas only 4 strains $(\sim 5 \%)$ carried the LEE marker gene eae [32]. In addition, a number of STEC strains of serotype O113:H21 were isolated during this study, a serotype which is highly correlated with severe diseases in humans [54]. One of these STEC O113:H21 strains, TS18/08, was eae-negative, but carried $s t x_{2}$ and EHEC-hlyA as well as the two toxin-encoding genes $s u b A B$ (subtilase cytotoxin) and $c d t-V$ (cytolethal distending toxin $\mathrm{V}$ ) and was therefore be considered as a potential human pathogen.

Similar results, regarding the prevalence of EHEC-hly $A$, were obtained by Feng and Reddy by analyzing 132 STEC strains isolated from fresh produce [55]. While EHEC-hlyA was detected in $61 \%$ of the isolates, only about $9 \%$ of the isolates were found to harbour the eae gene. Similar to the findings mentioned above, these results demonstrate once more that EHEC-hlyA is very common among STEC strains found in risk foods [55]. This fact was further confirmed by Boczek et al. [56], during the screening of effluent samples of municipal wastewater treatment plants for the presence of EHEC-hlyA. In total, $338 \mathrm{E}$. coli isolates were found to harbor the EHEC-hlyA gene, while only 2 isolates were shown to express the eae gene. Interestingly, neither $s t x_{1}$, nor $s t x_{2}$ could be detected in any of the samples. Subsequent serotyping of the isolates revealed serotypes, which were described previously to cause non-O157:H7 EHEC infections in humans, as well as serotypes which have never been reported in association with human infections. These results showed that EHEC-hlyA is widely distributed among E. coli isolates in the environment but is not automatically an indication for the presence of EHEC [56].

Askari Badouei et al. investigated the occurrence of EHEC-Hly in different diarrheagenic E coli strains isolated from cattle and evaluated the association with other virulence markers [57]. Among the 54 EHEC-hlyA-positive isolates $(\sim 12 \%), 25$ different virulence profiles were observed, reflecting a significant heterogeneity. EHEC-hlyA was mostly found in EHEC (72\%), but the toxin was also present in enteropathogenic E. coli (EPEC) with a percentage of $7.4 \%$. In addition, two potential hybrid EHEC-hlyA-positive pathotypes were found consisting of EPEC/ETEC or EHEC/ETEC. Similar to the results described above, these findings indicate that E. coli strains isolated from cattle, which are EHEC-hlyA-positive, may be genetically considerably heterogenous and may also belong to different pathotypes.

During a study with more than 400 STEC and non-STEC isolates from animal, food, environmental, and clinical sources, Lorenz et al. [11] investigated the prevalence and subtype patterns of EHEC-hlyA. 
The results obtained by PCR and RFLP revealed 301 EHEC-hlyA-positive samples ( 69\%), as well as six closely related subtypes (A to F), whereby subtypes A and D were found in eae-negative STEC and subtypes B, C, E, and F in eae-positive STEC strains. Furthermore, EHEC-hlyA subtypes differed significantly between isolates obtained from different sources: In animal isolates mostly subtypes A and $C$ were found, in food isolates mainly subtype A was detected, and clinical isolates contained mainly subtype C. Interestingly, only four strains carrying subtype D and two strains carrying subtype E were identified within this study, which were exclusively found in food or clinical isolates, respectively. In addition, this study revealed that certain O serogroups correlated with specific EHEC-hlyA subtypes: Subtype A with O104, O113, and O8; subtype B exclusively with O157; and subtype C with O26, O111, and O121 [11].

In a later study, Lorenz and colleagues further analyzed the plasmid containing the rare EHEC-hly $A$ subtypes D and E to elucidate the evolutionary relationship among different EHEC-hlyA subtypes and to examine their potential role in bacterial virulence [58]. The results showed that the plasmid carrying subtype D was exceptionally large (>200 kbp) and harbored different virulence genes, associated with EHEC as well as with enterotoxigenic E. coli (ETEC), although this subtype was only detected in food isolates. The authors therefore assumed that the plasmid carrying subtype D EHEC-hlyA represents a novel virulence plasmid, which has evolved from a different evolutionary lineage unlike the plasmids that carry other EHEC-hlyA subtypes. In contrast, the subtype E plasmid was smaller in size (50 kbp) and carried only a few virulence determinants even though this subtype was isolated from clinical samples associated with HUS. These findings led the authors suggest that the pathogenicity of this strains might be a result of chromosomally encoded virulence factors, particularly as subtype E isolates were all eae-positive STEC strains [58].

In a recent study, Fu et al. [59] investigated the occurrence and genetic diversity of the EHEC-hlyA gene in more than 400 non-O157 STEC isolates obtained from human, animal, and food in China, collected in different geographical regions [59]. The results demonstrated that EHEC-hlyA was present in $31.8 \%$ of the non-O157 STEC strains, a percentage which was lower than that found, for example, in a comparable study of Cookson et al. [17] where EHEC-hlyA was found in $63.8 \%$ of E. coli isolates obtained from cattle and sheep and $96 \%$ of isolates from patients with diarrhea. The authors therefore concluded that the prevalence of EHEC-hlyA might be different among various geographical locations and sources. Table 1 summarizes the prevalence of EHEC-hlyA in different samples origins. Due to sequence analysis of the detected EHEC-hlyA genes, Fu et al. [59] determined three different phylogenetic groups which are corresponding to that reported previously by Cookson et al. [17]. Fu and colleagues also demonstrated a clear relationship between EHEC-hlyA groups and their sources, similar to the findings of Lorenz et al. [11].

Table 1. Prevalence of EHEC-hlyA obtained from different sources.

\begin{tabular}{lll}
\hline \multicolumn{1}{c}{ Sample Origin } & $\begin{array}{l}\text { Percentage of EHEC- } \boldsymbol{h l y} \boldsymbol{A} \\
\text { Positive Samples }\end{array}$ & Reference \\
\hline E. coli isolates from normal cattle & $<1$ & {$[52]$} \\
STEC isolates from normal cattle & $36 \%$ & {$[53]$} \\
STEC isolates from risk foods & $40 \%$ & {$[32]$} \\
STEC isolates from fresh produce & $61 \%$ & {$[55]$} \\
E. coli isolates from effluent samples & 338 positive samples * & {$[56]$} \\
Diarrheagenic E. coli strains from cattle & $12 \%$ & {$[57]$} \\
STEC and non-STEC isolates from human, animal, & $69 \%$ & {$[11]$} \\
and food & & {$[59]$} \\
non-O157 STEC isolates from human, animal, & $31.8 \%$ & {$[17]$} \\
and food & $63.8 \%$ & {$[17]$} \\
E. coli isolates obtained from cattle and sheep & $96 \%$ & \\
STEC isolates from patients with diarrhea. &
\end{tabular}

* total no. of samples was not described in [56]. 


\section{Conclusions}

Although the precise role of EHEC-Hly in the pathogenesis of EHEC and other virulent E. coli strains is yet not fully understood, the toxin is recognized as a potential virulence factor and a commonly used virulence marker for the analysis of EHEC and STEC strains. The EHEC-hlyA gene is found very frequently in STEC isolates which belong to serotypes associated with severe human diseases. However, the clinical importance of this toxin alone is still unclear, as the association with either HC or HUS cannot be clearly assigned. This indicates that the clinical effect of EHEC-Hly might be very complex and depends on the correlation with several other virulence factors. Furthermore, EHEC-Hly is also present in environmental E. coli isolates, which are lacking Stx and thus should only be used in combination with other virulence factors for an estimation of the bacterial virulence potential. Further studies are necessary to investigate the role of EHEC-Hly in combination with Stx and other virulence factors for the development of EHEC-associated diseases. This could be achieved in several models using modern mutagenesis techniques such as CRISPR/Cas in combination with appropriate cell cultures and organoid models. Although the mode of pore-formation has been elucidated, more in depth research should be conducted on the mode of action and target structures. Moreover, the interaction with global regulators and their influence on EHEC-hlyA expression should be investigated further to elucidate the clinical importance of EHEC-Hly. In addition, the characterization of different EHEC-hlyA-subtypes could be a method for surveillance and documentation of EHEC infections, as a clear association between different subtypes and sample origins, as well as O serotypes, was shown.

Author Contributions: M.S. conceived, designed and wrote the manuscript; L.H. conceived and carried out the phenotypical EHEC hemolysin experiment shown in Figure 1, corrected and was included in proofreading the manuscript; H.S. was included in writing, correcting, and proofreading the manuscript.

Funding: This research received no external funding.

Conflicts of Interest: The authors declare no conflict of interest.

\section{References}

1. Beutin, L.; Montenegro, M.; Zimmermann, S.; Stephan, R. Characterization of hemolytic strains of Escherichia coli belonging to classical enteropathogenic O-serogroups. Zent. fur Bakteriol. Mikrobiol. und Hyg. 1986, 261, 266-279. [CrossRef]

2. Beutin, L.; Prada, J.; Zimmermann, S.; Stephan, R.; Ørskov, I.; Ørskov, F. Enterohemolysin, a new type of hemolysin produced by some strains of enteropathogenic E. coli (EPEC). Zent. fur Bakteriol. Mikrobiol. und Hyg. 1988, 267, 576-588. [CrossRef]

3. Beutin, L.; Montenegro, M.A.; Orskov, I.; Orskov, F.; Prada, J.; Zimmermann, S.; Stephan, R. Close association of verotoxin (Shiga-like toxin) production with enterohemolysin production in strains of Escherichia coli. J. Clin. Microbiol. 1989, 27, 2559-2564. [PubMed]

4. Schmidt, H.; Benz, R. Detection and characterization of EHEC-hemolysin. In E. Coli: Methods in Molecular Medicine; Philpott, D., Ebel, F., Eds.; Humana Press: Totowa, NJ, USA, 2003; pp. 151-163.

5. Beutin, L.; Stroeher, U.H.; Manning, P.A. Isolation of enterohemolysin (Ehly2)-associated sequences encoded on temperate phages of Escherichia coli. Gene 1993, 132, 95-99. [CrossRef]

6. Schmidt, H.; Karch, H.; Beutin, L. The large-sized plasmids of enterohemorrhagic Escherichia coli O157 strains encode hemolysins which are presumably members of the E. coli alpha-hemolysin family. FEMS Microbiol. Lett. 1994, 117, 189-196. [CrossRef]

7. O'Brien, A.D.; Lively, T.A.; Chang, T.W.; Gorbach, S.L. Purification of Shigella dysenteriae 1 (Shiga)-like toxin from Escherichia coli O157:H7 strain associated with haemorrhagic colitis. Lancet 1983, 8349, 573. [CrossRef]

8. Tzipori, S.; Gibson, R.; Montanaro, J. Nature and distribution of mucosal lesions associated with enteropathogenic and enterohemorrhagic Escherichia coli in piglets and the role of plasmid-mediated factors. Infect. Immun. 1989, 57, 1142-1150. [PubMed] 
9. Knutton, S.; Baldwin, T.; Williams, P.H.; McNeish, A.S. Actin accumulation at sites of bacterial adhesion to tissue culture cells: Basis of a new diagnostic test for enteropathogenic and enterohemorrhagic Escherichia coli. Infect. Immun. 1989, 57, 1290-1298.

10. Schmidt, H.; Karch, H. Enterohemolytic phenotypes and genotypes of Shiga toxin-producing Escherichia coli $\mathrm{O} 111$ strains from patients with diarrhea and hemolytic- uremic syndrome. J. Clin. Microbiol. 1996, 34, 2364-2367.

11. Lorenz, S.C.; Son, I.; Maounounen-Laasri, A.; Lin, A.; Fischer, M.; Kase, J.A. Prevalence of hemolysin genes and comparison of $e h x A$ subtype patterns in Shiga toxin-producing Escherichia coli (STEC) and non-STEC strains from clinical, food, and animal sources. Appl. Environ. Microbiol. 2013, 79, 6301-6311. [CrossRef]

12. Schmidt, H.; Beutin, L.; Karch, H. Molecular analysis of the plasmid-encoded hemolysin of Escherichia coli O157:H7 strain EDL 933. Infect. Immun. 1995, 63, 1055-1061. [PubMed]

13. Ludwig, A.; Schmid, A.; Benz, R.; Goebel, W. Mutations affecting pore formation by haemolysin from Escherichia coli. MGG Mol. Gen. Genet. 1991, 226, 198-208. [CrossRef] [PubMed]

14. Schmidt, H.; Kernbach, C.; Karch, H. Analysis of the EHEC hly operon and its location in the physical map of the large plasmid of enterohaemorrhagic Escherichia coli O157:H7. Microbiology 1996, 142, 907-914. [CrossRef] [PubMed]

15. Bauer, M.E.; Welch, R.A. Characterization of an RTX toxin from enterohemorrhagic Escherichia coli O157:H7. Infect. Immun. 1996, 64, 167-175. [PubMed]

16. Boerlin, P.; Chen, S.; Colbourne, J.K.; Johnson, R.; De Grandis, S.; Gyles, C. Evolution of enterohemorrhagic Escherichia coli hemolysin plasmids and the locus for enterocyte effacement in Shiga toxin-producing E. coli. Infect. Immun. 1998, 66, 2553-2561. [PubMed]

17. Cookson, A.L.; Bennett, J.; Thomson-Carter, F.; Attwood, G.T. Molecular subtyping and genetic analysis of the enterohemolysin gene $(e h x A)$ from Shiga toxin-producing Escherichia coli and atypical enteropathogenic E. coli. Appl. Environ. Microbiol. 2007, 73, 6360-6369. [CrossRef] [PubMed]

18. Newton, H.J. Shiga toxin-producing Escherichia coli strains negative for locus of enterocyte effacement. Emerging. Infect. Dis. 2009, 15, 372-380. [CrossRef]

19. Elliott, S.J.; Sperandio, V.; Giron, J.A.; Shin, S.; Mellies, J.L.; Wainwright, L.; Hutcheson, S.W.; McDaniel, T.K.; Kaper, J.B. The locus of enterocyte effacement (LEE)-encoded regulator controls expression of both LEEand non-LEE-encoded virulence factors in enteropathogenic and enterohemorrhagic Escherichia coli. Infect. Immun. 2000, 68, 6115-6126. [CrossRef]

20. Mellies, J.L.; Barron, A.M.S.; Carmona, A.M. Enteropathogenic and enterohemorrhagic Escherichia coli virulence gene regulation. Infect. Immun. 2007, 75, 4199-4210. [CrossRef]

21. Deng, W.; Puente, J.L.; Gruenheid, S.; Li, Y.; Vallance, B.A.; Vazquez, A.; Barba, J.; Ibarra, J.A.; O’Donnell, P.; Metalnikov, P.; et al. Dissecting virulence: Systematic and functional analyses of a pathogenicity island. Proc. Natl. Acad. Sci. USA 2004, 101, 3597-3602. [CrossRef]

22. Saitoh, T.; Iyoda, S.; Yamamoto, S.; Lu, Y.; Shimuta, K.; Ohnishi, M.; Terajima, J.; Watanabe, H. Transcription of the ehx enterohemolysin gene is positively regulated by GrlA, a global regulator encoded within the locus of enterocyte effacement in enterohemorrhagic Escherichia coli. J. Bacteriol. 2008, 190, 4822-4830. [CrossRef] [PubMed]

23. Iyoda, S.; Honda, N.; Saitoh, T.; Shimuta, K.; Terajima, J.; Watanabe, H.; Ohnishi, M. Coordinate control of the locus of enterocyte effacement and enterohemolysin genes by multiple common virulence regulators in enterohemorrhagic Escherichia coli. Infect. Immun. 2011, 79, 4628-4637. [CrossRef] [PubMed]

24. Honda, N.; Iyoda, S.; Yamamoto, S.; Terajima, J.; Watanabe, H. LrhA positively controls the expression of the locus of enterocyte effacement genes in enterohemorrhagic Escherichia coli by differential regulation of their master regulators PchA and PchB. Mol. Microbiol. 2009, 74, 1393-1441. [CrossRef] [PubMed]

25. Li, H.; Granat, A.; Stewart, V.; Gillespie, J.R. RpoS, H-NS, and DsrA influence EHEC hemolysin operon (ehxCABD) transcription in Escherichia coli O157:H7 strain EDL933. FEMS Microbiol. Lett. 2008, 285, 257-262. [CrossRef] [PubMed]

26. Sledjeski, D.D.; Gupta, A.; Gottesman, S. The small RNA, DsrA, is essential for the low temperature expression of RpoS during exponential growth in Escherichia coli. EMBO J. 1996, 15, 3993-4000. [CrossRef] [PubMed]

27. Majdalani, N.; Cunning, C.; Sledjeski, D.; Elliott, T.; Gottesman, S. DsrA RNA regulates translation of RpoS message by an anti-antisense mechanism, independent of its action as an antisilencer of transcription. Proc. Natl. Acad. Sci. USA 2002, 95, 12462-12467. [CrossRef] [PubMed] 
28. Laaberki, M.H.; Janabi, N.; Oswald, E.; Repoila, F. Concert of regulators to switch on LEE expression in enterohemorrhagic Escherichia coli O157:H7: Interplay between Ler, GrlA, HNS and RpoS. Int. J. Med. Microbiol. 2006, 296, 197-210. [CrossRef]

29. Hengge-Aronis, R. Signal transduction and regulatory mechanisms involved in control of the oS (RpoS) subunit of RNA Polymerase. Microbiol. Mol. Biol. Rev. 2003, 66, 373-395. [CrossRef]

30. Barth, M.; Marschall, C.; Muffler, A.; Fischer, D.; Hengge-Aronis, R. Role for the histone-like protein H-NS in growth phase-dependent and osmotic regulation of $\sigma(\mathrm{S})$ and many $\sigma(\mathrm{S})$-dependent genes in Escherichia coli. J. Bacteriol. 1995, 177, 3455-3464. [CrossRef]

31. Rogers, M.T.; Zimmerman, R.; Scott, M.E. Histone-like nucleoid-structuring protein represses transcription of the ehx operon carried by locus of enterocyte effacement-negative Shiga toxin-expressing Escherichia coli. Microb. Pathog. 2009, 47, 202-211. [CrossRef]

32. Slanec, T.; Fruth, A.; Creuzburg, K.; Schmidt, H. Molecular analysis of virulence profiles and Shiga toxin genes in food-borne Shiga toxin-producing Escherichia coli. Appl. Environ. Microbiol. 2009, 75, 6187-6197. [CrossRef] [PubMed]

33. Heinisch, L.; Zoric, K.; Krause, M.; Schmidt, H. Transcription of the subtilase cytotoxin gene subAB 1 in Shiga toxin-producing Escherichia coli is dependent on $h f q$ and hns. Appl. Environ. Microbiol. 2019, AEM.01281-19. [CrossRef] [PubMed]

34. Schmidt, H.; Maier, E.; Karch, H.; Benz, R. Pore-forming properties of the plasmid-encoded hemolysin of enterohemorrhagic Escherichia coli 0157: H7. Eur. J. Biochem. 1996, 241, 594-601. [CrossRef] [PubMed]

35. Bhakdi, S.; Mackman, N.; Nicaud, J.M.; Holland, I.B. Escherichia coli hemolysin may damage target cell membranes by generating transmembrane pores. Infect. Immun. 1986, 52, 63-69. [PubMed]

36. Taneike, I.; Zhang, H.-M.; Wakisaka-Saito, N.; Yamamoto, T. Enterohemolysin operon of Shiga toxin-producing Escherichia coli: A virulence function of inflammatory cytokine production from human monocytes. FEBS Lett. 2002, 524, 219-224. [CrossRef]

37. Zhang, X.; Cheng, Y.; Xiong, Y.; Ye, C.; Zheng, H.; Sun, H.; Zhao, H.; Ren, Z.; Xu, J. Enterohemorrhagic Escherichia coli specific enterohemolysin induced IL-1 $\beta$ in human macrophages and EHEC-induced IL-1 $\beta$ required activation of NLRP3 inflammasome. PLOS ONE 2012, 7, e50288. [CrossRef]

38. Van de Kar, N.C.; Monnens, L.A.; Karmali, M.A.; van Hinsbergh, V.W. Tumor necrosis factor and interleukin-1 induce expression of the verocytotoxin receptor globotriaosylceramide on human endothelial cells: Implications for the pathogenesis of the hemolytic uremic syndrome. Blood 1992, 80, 2755-2764.

39. Aldick, T.; Bielaszewska, M.; Zhang, W.; Brockmeyer, J.; Schmidt, H.; Friedrich, A.W.; Kim, K.S.; Schmidt, M.A.; Karch, H. Hemolysin from Shiga toxin-negative Escherichia coli O26 strains injures microvascular endothelium. Microbes Infect. 2007, 9, 282-290. [CrossRef]

40. Aldick, T.; Bielaszewska, M.; Uhlin, B.E.; Humpf, H.-U.; Wai, S.N.; Karch, H. Vesicular stabilization and activity augmentation of enterohaemorrhagic Escherichia coli haemolysin. Mol. Microbiol. 2009, 71, 1496-1508. [CrossRef]

41. Bielaszewska, M.; Aldick, T.; Bauwens, A.; Karch, H. Hemolysin of enterohemorrhagic Escherichia coli: Structure, transport, biological activity and putative role in virulence. Int. J. Med. Microbiol. 2014, 304, 521-529. [CrossRef]

42. Kuehn, M.J. Bacterial outer membrane vesicles and the host-pathogen interaction. Genes Dev. 2005, 19, 2645-2655. [CrossRef] [PubMed]

43. Bauer, M.E.; Welch, R.A. Association of RTX toxins with erythrocytes. Infect. Immun. 1996, 64, 4665-4672. [PubMed]

44. Bielaszewska, M.; Rüter, C.; Kunsmann, L.; Greune, L.; Bauwens, A.; Zhang, W.; Kuczius, T.; Kim, K.S.; Mellmann, A.; Schmidt, M.A.; et al. Enterohemorrhagic Escherichia coli hemolysin employs outer membrane vesicles to target mitochondria and cause endothelial and epithelial apoptosis. PLoS Pathog. 2013, 9, e1003797. [CrossRef] [PubMed]

45. Bielaszewska, M.; Rüter, C.; Bauwens, A.; Greune, L.; Jarosch, K.A.; Steil, D.; Zhang, W.; He, X.; Lloubes, R.; Fruth, A.; et al. Host cell interactions of outer membrane vesicle-associated virulence factors of enterohemorrhagic Escherichia coli O157: Intracellular delivery, trafficking and mechanisms of cell injury. PLoS Pathog. 2017, 13, e1006159. [CrossRef] [PubMed] 
46. Beutin, L.; Zimmermann, S.; Gleier, K. Rapid detection and isolation of Shiga-like toxin (Verocytotoxin)producing Escherichia coli by direct testing of individual enterohemolytic colonies from washed sheep blood agar plates in the VTEC-RPLA assay. J. Clin. Microbiol. 1996, 34, 2812-2814. [PubMed]

47. Lehmacher, A.; Meier, H.; Aleksic, S.; Bockemühl, J. Detection of hemolysin variants of Shiga toxin-producing Escherichia coli by PCR and culture on vancomycin-cefixime-cefsulodin blood agar. Appl. Environ. Microbiol. 1998, 64, 2449-2453. [PubMed]

48. Bai, X.; Mernelius, S.; Jernberg, C.; Einemo, I.-M.; Monecke, S.; Ehricht, R.; Löfgren, S.; Matussek, A. Shiga toxin-producing Escherichia coli infection in Jönköping County, Sweden: Occurrence and molecular characteristics in correlation with clinical symptoms and duration of Stx shedding. Front. Cell. Infect. Microbiol. 2018, 8, 125. [CrossRef]

49. Ethelberg, S.; Olsen, K.E.P.; Scheutz, F.; Jensen, C.; Schiellerup, P.; Engberg, J.; Petersen, A.M.; Olesen, B.; Gerner-Smidt, P.; Mølbak, K. Virulence factors for hemolytic uremic syndrome, Denmark. Emerg. Infect. Dis. 2004, 10, 842-847. [CrossRef]

50. Patel, I.R.; Gangiredla, J.; Lacher, D.W.; Mammel, M.K.; Bagi, L.; Baranzoni, G.M.; Fratamico, P.M.; Roberts, E.L.; Debroy, C.; Lindsey, R.L. Interlaboratory evaluation of the U.S. Food and Drug Administration Escherichia coli identification microarray for profiling Shiga toxin-producing Escherichia coli. J. Food Prot. 2018, 81, 1275-1282. [CrossRef]

51. Shridhar, P.B.; Patel, I.R.; Gangiredla, J.; Noll, L.W.; Shi, X.; Bai, J.; Elkins, C.A.; Strockbine, N.; Nagaraja, T.G. DNA microarray-based assessment of virulence potential of Shiga toxin gene-carrying Escherichia coli O104:H7 isolated from feedlot cattle feces. PLoS ONE 2018, 13, e0196490. [CrossRef]

52. Gyles, C.; Johnson, R.; Gao, A.; Ziebell, K.; Pierard, D.; Aleksic, S.; Boerlin, P. Association of enterohemorrhagic Escherichia coli hemolysin with serotypes of Shiga-like-toxin-producing Escherichia coli of human and bovine origins. Appl. Environ. Microbiol. 1998, 64, 4134-4141. [PubMed]

53. Sandhu, K.S.; Clarke, R.C.; Gyles, C.L. Virulence markers in Shiga toxin-producing Escherichia coli isolated from cattle. J. Vet. Res. 1999, 63, 177-184.

54. Hussein, H.S. Prevalence and pathogenicity of Shiga toxin-producing Escherichia coli in beef cattle and their products. J. Anim. Sci. 2007, 85, E63-E72. [CrossRef] [PubMed]

55. Feng, P.C.H.; Reddy, S. Prevalences of Shiga toxin subtypes and selected other virulence factors among Shiga-toxigenic Escherichia coli strains isolated from fresh produce. Appl. Environ. Microbiol. 2013, 79, 6917-6923. [CrossRef] [PubMed]

56. Boczek, L.A.; Johnson, C.H.; Rice, E.W.; Kinkle, B.K. The widespread occurrence of the enterohemolysin gene ehlyA among environmental strains of Escherichia coli. FEMS Microbiol. Lett. 2006, 254, 281-284. [CrossRef] [PubMed]

57. Askari Badouei, M.; Morabito, S.; Najafifar, A.; Mazandarani, E. Molecular characterization of enterohemorrhagic Escherichia coli hemolysin gene (EHEC-hlyA)-harboring isolates from cattle reveals a diverse origin and hybrid diarrheagenic strains. Infect. Genet. Evol. 2016, 39, 342-348. [CrossRef] [PubMed]

58. Lorenz, S.C.; Monday, S.R.; Hoffmann, M.; Fischer, M.; Kase, J.A. Plasmids from Shiga toxin-producing Escherichia coli strains with rare enterohemolysin gene (ehxA) subtypes reveal pathogenicity potential and display a novel evolutionary path. Appl. Environ. Microbiol. 2016, 82, 6367-6377. [CrossRef] [PubMed]

59. Fu, S.; Bai, X.; Fan, R.; Sun, H.; Xu, Y.; Xiong, Y. Genetic diversity of the enterohaemolysin gene $(e h x A)$ in non-O157 Shiga toxin-producing Escherichia coli strains in China. Sci. Rep. 2018, 8, 1-8. [CrossRef] [PubMed] 